\title{
Adaptive Stabilization of Stochastic Nonlinear Systems Disturbed by Unknown Time Delay and Covariance Noise
}

\author{
Na Duan, ${ }^{1}$ Huifang Min, ${ }^{2}$ and Xiaoyan Qin ${ }^{3}$ \\ ${ }^{1}$ School of Electrical Engineering \& Automation, Jiangsu Normal University, Xuzhou 221116, China \\ ${ }^{2}$ School of Automation, Nanjing University of Science and Technology, Nanjing 210094, China \\ ${ }^{3}$ School of Mathematics and Statistics, Zaozhuang University, Zaozhuang 277160, China \\ Correspondence should be addressed to Na Duan; duanna08@163.com
}

Received 6 March 2017; Accepted 2 April 2017; Published 30 April 2017

Academic Editor: Weihai Zhang

Copyright (c) $2017 \mathrm{Na}$ Duan et al. This is an open access article distributed under the Creative Commons Attribution License, which permits unrestricted use, distribution, and reproduction in any medium, provided the original work is properly cited.

\begin{abstract}
This paper considers a more general stochastic nonlinear time-delay system driven by unknown covariance noise and investigates its adaptive state-feedback control problem. As a remarkable feature, the growth assumptions imposed on delay-dependent nonlinear terms are removed. Then, with the help of Lyapunov-Krasovskii functionals and adaptive backstepping technique, an adaptive statefeedback controller is constructed by overcoming the negative effects brought by unknown time delay and covariance noise. Based on the designed controller, the closed-loop system can be guaranteed to be globally asymptotically stable (GAS) in probability. Finally, a simulation example demonstrates the effectiveness of the proposed scheme.
\end{abstract}

\section{Introduction}

In control fields, stochastic noises (white noise, Levy noise, etc.) extensively occur in real plants including parameter perturbations, stochastic errors, and external environment variations. Therefore, the investigation of stochastic nonlinear systems is meaningful both theoretically and practically. During the past decades, the backstepping technique presented by [1] for stochastic nonlinear systems has been proven to be an effective design tool. Based on the backstepping technique, Lyapunov function method, and stochastic stability theory, recent years have witnessed considerable results on stochastic nonlinear systems; see [2-17] and the references therein. Particularly, adaptive backstepping technique, a recursive design procedure, has been extended to stochastic nonlinear systems with various uncertainties and many significant developments have been achieved in [9-17].

As is well-known time delays frequently exist in practical systems such as electrical networks, microwave oscillator, and chemical reactor systems. The existence of time delays may deteriorate system performance and cause instability. Therefore, the control and design for stochastic nonlinear time-delay systems has been one of the active research topics and already obtained fruitful results [18-33]. In [18-22], by using Lyapunov-Krasovskii functional method, the outputfeedback stabilization problems were solved for stochastic nonlinear systems with time delays only presenting in system output. References $[23,24]$ considered the control problems of high-order stochastic nonlinear time-delay systems by introducing the adding a power integrator technique. However, the growth conditions assumed on system nonlinearities somewhat restrict the extension of the proposed control schemes in [18-24].

In recent years, how to weaken or remove the traditional nonlinear growth assumptions has been the main focus and difficulty in stochastic nonlinear time-delay systems control. In [25-27], the homogeneous domination approach was extended to stochastic nonlinear time-delay systems and the assumptions on nonlinearities in drift and diffusion vector fields were relaxed. In addition, the adaptive control technique has been applied with neural network approximation approach to weaken the assumptions on system time-delay nonlinearities in [28-32] and the related references. Recently, the traditional pure growth assumptions were further relaxed for stochastic nonlinear time-delay systems in [33] with the help of parameter-based controller design method. Despite 
the remarkable efforts obtained on relaxing the growth assumptions, the existing results including [25-33] all failed to remove these nonlinear growth assumptions.

On the other hand, it is well-known that the noise of unknown covariance is also a source of uncertainties, which may bring some negative effects on systems. In the past decades, the control problems for stochastic nonlinear systems driven by noise of unknown covariance have been studied in [34-36] by using Lyapunov functions and stochastic stability theorem. However, to the best of the authors' knowledge, for stochastic nonlinear time-delay systems driven by unknown covariance noise, there are few related results. Motivated by the aforementioned discussions, a natural problem arises:

How to remove the growth assumptions on system nonlinearities and further stabilize stochastic nonlinear time-delay systems driven by unknown covariance noise?

This paper will focus on handling the above problem. The main contributions are listed as follows: (i) this paper considers a more general class of stochastic nonlinear systems disturbed by both unknown time delay and covariance noise. A distinctive novelty is that the growth assumptions imposed on time-delay nonlinearities in existing results are proven to be unnecessary and can be removed. (ii) By utilizing adaptive control technique and Lyapunov-Krasovskii functional method, the adverse effects brought by unknown covariance noise and time delay are compensated and an adaptive statefeedback controller is designed. It is proven that the designed controller can render the closed-loop system globally asymptotically stable (GAS) in probability.

The remainder of this paper is organized as follows. Section 2 gives the mathematical preliminaries. The design process and analysis procedure are given in Sections 3 and 4, respectively. In Section 5, a simulation example is presented. Section 6 concludes this paper. Some necessary proof is provided in Appendix.

\section{Mathematical Preliminaries}

The following notations, definition, and lemmas will be used throughout the whole paper.

Notations. $\mathbb{R}^{+}$denotes the set of all the nonnegative real numbers; $\mathbb{R}^{i}$ denotes the $i$-dimensional Euclidean space; $\mathscr{C}^{i}$ denotes the family of all the functions with continuous $i$ th partial derivations; $|\cdot|$ denotes the Euclidean norm of a vector or a square matrix; $X^{T}$ denotes the transpose of a given vector or matrix $X$ and $\operatorname{Tr}\{X\}$ denotes its trace when $X$ is square; $\mathscr{C}\left([-d, 0] ; \mathbb{R}^{n}\right)$ denotes the space of continuous $\mathbb{R}^{n}$ valued functions on $[-d, 0]$ endowed with the norm $\|\cdot\|$ defined by $\|f\|=\sup _{x \in[-d, 0]}|f(x)|$ for $f \in \mathscr{C}\left([-d, 0], \mathbb{R}^{n}\right)$; $\mathscr{C}_{\mathscr{F}_{0}}^{b}\left([-d, 0], \mathbb{R}^{n}\right)$ stands for the family of all $\mathscr{F}_{0}$-measurable bounded $\mathscr{C}\left([-d, 0] ; \mathbb{R}^{n}\right)$-valued random variables $\xi=\{\xi(\theta)$ : $-d \leq \theta \leq 0\} ; \mathscr{K}$ denotes the set of all functions: $\mathbb{R}^{+} \rightarrow$ $\mathbb{R}^{+}$, which are continuous, strictly increasing, and vanishing at zero; $\mathscr{K}_{\infty}$ denotes the set of all functions which are of class $\mathscr{K}$ and unbounded; $\mathscr{K} \mathscr{L}$ is the set of all functions $\beta(s, t): \mathbb{R}^{+} \times \mathbb{R}^{+} \rightarrow \mathbb{R}^{+}$, which are of $\mathscr{K}$ for each fixed $t$ and decrease to zero as $t \rightarrow \infty$ for each fixed $s$.

Consider stochastic nonlinear time-delay system

$$
\begin{array}{r}
d x(t)=f(x(t), x(t-d)) d t+g(x(t), x(t-d)) d \omega, \\
\forall t \geq 0,
\end{array}
$$

where the initial data is $x(\theta)=\xi$ for $-d \leq \theta \leq 0$; $d>0$ is a constant delay; $\omega$ is an $r$-dimensional standard wiener process defined on a complete probability space $\{\Omega, \mathscr{F}, P\}$, where $\Omega$ is a sample space, $\mathscr{F}$ is a $\sigma$-field, and $P$ is the probability measure with a natural filtration $\{\mathscr{F}\}_{t \geq 0}$ (i.e., $\mathscr{F}_{t}=$ $\sigma\{w(s): 0 \leq s \leq t\})$; the drift term $f: \mathbb{R}^{n} \times \mathbb{R}^{n} \rightarrow \mathbb{R}^{n}$ and the diffusion term $g: \mathbb{R}^{n} \times \mathbb{R}^{n} \rightarrow \mathbb{R}^{n \times r}$ are locally Lipschitz functions with $f(0,0)=0$ and $g(0,0)=0$. Obviously, system (1) admits a trivial solution $x(0)=0$. For any given $V(x(t)) \epsilon$ $C^{2}$, the differential operator $\mathscr{L}$ along system (1) is defined as

$$
\mathscr{L} V=\frac{\partial V}{\partial x} f+\frac{1}{2} \operatorname{Tr}\left\{g^{T} \frac{\partial^{2} V}{\partial x^{2}} g\right\}
$$

Definition 1 (see [22]). The equilibrium $x=0$ of system (1) is said to be globally asymptotically stable (GAS) in probability if for any $\epsilon>0$, there exists a function $\beta(\cdot, \cdot) \in \mathscr{K} \mathscr{L}$ such that $P\{|x(t)| \leq \beta(\|\xi\|, t)\} \geq 1-\epsilon$ for any $t \geq 0$ and $\xi \epsilon$ $\mathscr{C}_{\mathscr{F}_{0}}^{b}\left([-d, 0], \mathbb{R}^{n}\right) \backslash\{0\}$, where $\|\xi\|=\sup _{\theta \in[-d, 0]}|x(\theta)|$.

Lemma 2 (see [22]). For system (1), if there exist functions $V(x(t)) \in \mathscr{C}^{2}$ and $\chi_{1}, \chi_{2} \in \mathscr{K}_{\infty}$ such that

$$
\begin{aligned}
& \chi_{1}(|x(t)|) \leq V(x(t)) \leq \chi_{2}\left(\sup _{-d \leq s \leq 0}|x(t+s)|\right) \\
& \mathscr{L} V(x(t)) \leq-W(x(t))
\end{aligned}
$$

when $W(x(t)) \in \mathscr{K}$, then there exists a unique solution on $[-d, \infty)$ for system (1) and the equilibrium $x=0$ is GAS in probability with $P\left\{\lim _{t \rightarrow \infty}|x(t)|=0\right\}=1$.

Lemma 3 (see [9]). For any smooth function $f(x), x \in R^{n}$, there exists a smooth function $\bar{f}(x)$ such that $f(x)-f(0)=$ $\left(\left.\int_{0}^{1}(\partial f(\lambda) / \partial \lambda)\right|_{\lambda=\alpha x} d \alpha\right) x=x \bar{f}(x)$.

Lemma 4 (see [37]). For any real numbers $x, y m, n>0$ and continuous function $a(\cdot) \geq 0, a(\cdot) x^{m} y^{n} \leq b|x|^{m+n}+(n /(m+$ $n))((m+n) / m)^{-m / n} a(\cdot)^{(m+n) / n} b^{-m / n}|y|^{m+n}$ holds with $b>0$ being a real design constant.

\section{State-Feedback Controller Design}

In this section, we first present the problem to be investigated and a key lemma used in the design procedure. Then, based on adaptive backstepping technique, the recursive design procedure is given to construct an adaptive state-feedback controller. 
3.1. Problem Formulation. In this paper, we consider the following stochastic nonlinear time-delay system:

$$
\begin{gathered}
d x_{i}=x_{i+1} d t+f_{i}\left(\bar{x}_{i}, \bar{x}_{i}(t-d)\right) d t+g_{i}\left(x_{1}\right) \Sigma d \omega, \\
i=1, \ldots, n-1, \\
d x_{n}=u d t+f_{n}(x, x(t-d)) d t+g_{n}\left(x_{1}\right) \Sigma d \omega,
\end{gathered}
$$

where $u \in \mathbb{R}$ and $x=\left(x_{1}, \ldots, x_{n}\right)^{T} \in \mathbb{R}^{n}$ are system control input and measurable states, respectively; $\bar{x}_{i}(t-d)=\left(x_{1}(t-\right.$ $\left.d), \ldots, x_{i}(t-d)\right)^{T}, \bar{x}_{i}=\left(x_{1}, \ldots, x_{i}\right)^{T}$, and $x(t-d)=\left(x_{1}(t-\right.$ $\left.d), \ldots, x_{n}(t-d)\right)^{T} ; d>0$ is a constant time delay; $\omega$ is defined as in (1); $\Sigma: \mathbb{R}^{+} \rightarrow \mathbb{R}^{r \times r}$ is an unknown bounded nonnegative definite Borel measurable matrix function and $\Sigma \Sigma^{T}$ denotes the infinitesimal covariance function of the driving noise $\sum d \omega$; for $i=1, \ldots, n$, the drift terms $f_{i}\left(\bar{x}_{i}, \bar{x}_{i}(t-d)\right)$ : $\mathbb{R}^{i} \times \mathbb{R}^{i} \rightarrow \mathbb{R}$ are $\mathscr{C}^{1}$ functions with $f_{i}(0,0)=0$ and the diffusion terms $g_{i}\left(x_{1}\right): \mathbb{R} \rightarrow \mathbb{R}^{1 \times r}$ are smooth functions with $g_{i}(0)=0$.

The control objective is to design an adaptive statefeedback controller to render system (5) to be GAS in probability by removing the growth assumptions on delaydependent $f_{i}$. To make this feasible, we first show a lemma, which plays a key role in designing the ideal controller.

Lemma 5. For $\mathscr{C}^{1}$ functions $f_{i}\left(\bar{x}_{i}, \bar{x}_{i}(t-d)\right), i=1, \ldots, n$, with $f_{i}(0,0)=0$, there exist nonnegative smooth functions $\rho_{i j}\left(\bar{x}_{j}\right)$ such that

$$
\left|f_{i}\left(\bar{x}_{i}, \bar{x}_{i}(t-d)\right)\right| \leq \sum_{j=1}^{i} \rho_{i j}\left(\bar{x}_{j}\right)\left(\left|x_{j}\right|+\left|x_{j}(t-d)\right|\right) .
$$

Proof. In terms of Lemma 2.5 in [38], it holds that

$$
\begin{aligned}
& \left|f_{i}\left(\bar{x}_{i}, \bar{x}_{i}(t-d)\right)\right| \\
& \quad \leq \sum_{j=1}^{i}\left(\lambda_{i j}\left(\bar{x}_{j}\right)\left|x_{j}\right|+\lambda_{i j}^{*}\left(\bar{x}_{j}(t-d)\right)\left|x_{j}(t-d)\right|\right),
\end{aligned}
$$

where $\lambda_{i j}\left(\bar{x}_{j}\right)$ and $\lambda_{i j}^{*}\left(\bar{x}_{j}(t-d)\right)$ are nonnegative smooth functions. Then, there must exist sufficiently large nonnegative smooth functions $\bar{\lambda}_{i j}\left(\bar{x}_{j}\right)$ such that $\lambda_{i j}^{*}\left(\bar{x}_{j}(t-d)\right) \leq \bar{\lambda}_{i j}\left(\bar{x}_{j}\right)$. Considering this with (7), then (6) can hold by choosing $\rho_{i j}\left(\bar{x}_{j}\right)=\max \left\{\lambda_{i j}, \bar{\lambda}_{i j}\right\}$.

In addition, for $i=1, \ldots, n$, since $g_{i}(0)=0$ and $g_{i}\left(x_{1}\right)$ are smooth, using Lemma 3, one gets

$$
g_{i}\left(x_{1}\right)=x_{1} \bar{g}_{i}\left(x_{1}\right),
$$

where $\bar{g}_{i}\left(x_{1}\right)(i=1, \ldots, n)$ are smooth functions.

Remark 6. For stochastic nonlinear time-delay systems, previous works such as [18-33] all gave the growth conditions similar to (6) through imposing assumptions on system nonlinearities. In this paper, Lemma 5 proves that these assumptions are unnecessary and can be removed, which is a main distinctive feature of this paper. In addition, we give an example to show Lemma 5 can hold. Considering $f_{2}\left(\bar{x}_{2}, \bar{x}_{2}(t-d)\right)=x_{1}^{2}+x_{2}^{2}+x_{1}(t-d) \sin \left(x_{1}(t-d)\right) x_{1}+$ $\sin \left(x_{1}\right) x_{2}(t-d)$, one gets $\lambda_{21}=x_{1}, \lambda_{21}^{*}=x_{1} \sin \left(x_{1}(t-d)\right)$, and $\lambda_{22}=x_{2}, \lambda_{22}^{*}=\sin \left(x_{1}\right)$. Then, it can be verified that $\bar{\lambda}_{21}=(1 / 2)\left(1+x_{1}^{2}\right)$ and $\bar{\lambda}_{22}=1+x_{1}^{2}$. Thus, Lemma 5 is satisfied with $\rho_{21}=\max \left\{\lambda_{21}, \bar{\lambda}_{21}\right\}$ and $\rho_{22}=\max \left\{\lambda_{22}, \bar{\lambda}_{22}\right\}$.

3.2. Design of State-Feedback Controller. Before giving the detailed design procedure, introduce the state coordinate transformation as

$$
\begin{aligned}
& z_{1}=x_{1}, \\
& z_{i}=x_{i}-\alpha_{i}\left(\bar{x}_{i-1}, \widehat{\theta}\right), \quad i=2, \ldots, n,
\end{aligned}
$$

where $\alpha_{2}, \ldots, \alpha_{n}$ are virtual control laws to be determined and $\widehat{\theta}$ is the estimate of $\theta$ with the form

$$
\theta \triangleq \max \left\{\left|\Sigma \Sigma^{T}\right|,\left|\Sigma \Sigma^{T}\right|^{2},\left|\Sigma \Sigma^{T}\right|^{4 / 3}\right\} .
$$

According to (5), (9), and Itô's differentiation formula, it is easy to get

$$
\begin{aligned}
d z_{i} & =\left(x_{i+1}+F_{i d}-\sum_{j=1}^{i-1} \frac{\partial \alpha_{i}}{\partial x_{j}} x_{j+1}\right. \\
& \left.-\frac{1}{2} \sum_{j, k=1}^{i-1} \frac{\partial^{2} \alpha_{i}}{\partial x_{j} \partial x_{k}} g_{j} \Sigma \Sigma^{T} g_{k}^{T}-\frac{\partial \alpha_{i}}{\partial \hat{\theta}}\right) d t+G_{i} \Sigma d w
\end{aligned}
$$

where $i=1, \ldots, n, F_{i d}$, and $f_{i d}$ denote $F_{i}\left(\bar{x}_{i}, \bar{x}_{i}(t-d)\right)$ and $f_{i}\left(\bar{x}_{i}, \bar{x}_{i}(t-d)\right)$, respectively; $F_{i d}=f_{i d}-\sum_{j=1}^{i-1}\left(\partial \alpha_{i} / \partial x_{j}\right) f_{j d}$ and $G_{i}=g_{i}-\sum_{j=1}^{i-1}\left(\partial \alpha_{i} / \partial x_{j}\right) g_{j}$.

In the sequel, we aim to give the adaptive design procedure by combining Lyapunov-Krasovskii functionals with backstepping. The detailed process is divided into $n$ steps.

Step 1. Consider Lyapunov function $V_{1}=(1 / 4) z_{1}^{4}+(1 / 2 \gamma) \widetilde{\theta}^{2}$ for system (5), where $\widetilde{\theta}=\theta-\widehat{\theta}$ is the estimate error of $\theta$ and $\gamma>0$ is the adaptive gain constant. Then, by means of (2), (8), (10)-(11), Lemmas 4 and 5, and Itô's rule, it can be verified that

$$
\begin{aligned}
\mathscr{L} V_{1}= & z_{1}^{3} x_{2}+z_{1}^{3} f_{1 d}+\frac{3}{2} z_{1}^{2} g_{1} \Sigma \Sigma^{T} g_{1}^{T}-\frac{\tilde{\theta}}{\gamma} \dot{\hat{\theta}} \\
\leq & z_{1}^{3} x_{2}+\left|z_{1}\right|^{3} \rho_{11}\left(x_{1}\right)\left(\left|x_{1}\right|+\left|x_{1}(t-d)\right|\right) \\
& +\frac{3}{2} z_{1}^{4}\left|\bar{g}_{1}\right|^{2} \theta-\frac{\tilde{\theta}}{\gamma} \dot{\hat{\theta}} \\
\leq & z_{1}^{3}\left(x_{2}-\alpha_{2}\right)+z_{1}^{3} \alpha_{2}+\rho_{11}\left(x_{1}\right) z_{1}^{4} \\
& +b_{11} z_{1}^{4}(t-d)+\phi_{11}\left(x_{1}\right) z_{1}^{4}+z_{1}^{4} \varphi_{1}\left(x_{1}\right) \hat{\theta} \\
& \quad-\frac{\tilde{\theta}}{\gamma}\left(\dot{\hat{\theta}}-\tau_{1}\right),
\end{aligned}
$$

where $\phi_{11}\left(x_{1}\right)=(3 / 4)\left(4 b_{11}\right)^{-1 / 3} \rho_{11}^{4 / 3}$ with $b_{11}>0$ being a design constant; $\varphi_{1}\left(x_{1}\right)=(3 / 2)\left|\bar{g}_{1}\right|^{2}$ and $\tau_{1}=\gamma \varphi_{1}\left(x_{1}\right) z_{1}^{4}$. 
Then, constructing the Lyapunov-Krasovskii functional

$$
V_{1 K}=V_{1}+W_{1}, \quad W_{1}=\int_{t-d}^{t} b_{11} z_{1}^{4}(s) d s
$$

and the first virtual control law

$$
\begin{aligned}
\alpha_{2}\left(x_{1}, \hat{\theta}\right)= & -z_{1} \beta_{1}\left(x_{1}, \hat{\theta}\right), \\
\beta_{1}\left(x_{1}, \hat{\theta}\right)= & c_{11}+\rho_{11}\left(x_{1}\right)+\phi_{11}\left(x_{1}\right)+\varphi_{1}\left(x_{1}\right) \hat{\theta} \\
& +b_{11},
\end{aligned}
$$

together with (2) and (12), one yields

$$
\mathscr{L} V_{1 K} \leq-c_{11} z_{1}^{4}+z_{1}^{3}\left(x_{2}-\alpha_{2}\right)-\frac{\tilde{\theta}}{\gamma}\left(\dot{\vec{\theta}}-\tau_{1}\right)
$$

where $c_{11}$ is a positive design constant.

Step $i(2 \leq i \leq n-1)$. We give the inductive step through a proposition.

Proposition 7. If at step $(i-1)$ there exist a series of virtual control laws $\alpha_{2}=-z_{1} \beta_{1}\left(x_{1}, \widehat{\theta}\right), \ldots, \alpha_{i}=-z_{i-1} \beta_{i-1}\left(\bar{x}_{i-1}, \widehat{\theta}\right)$ making the Lyapunov-Krasovskii functional $V_{i-1, K}=$ $(1 / 4) \sum_{j=1}^{i-1} z_{j}^{4}+\sum_{j=1}^{i-1} W_{j}+(1 / 2 \gamma) \widetilde{\theta}^{2}$ satisfy

$$
\begin{aligned}
\mathscr{L} V_{i-1, K} \leq & -\sum_{j=1}^{i-1} c_{j, i-1} z_{j}^{4}+z_{i-1}^{3}\left(x_{i}-\alpha_{i}\right) \\
& -\left(\frac{\widetilde{\theta}}{\gamma}+\sum_{j=2}^{i-1} z_{j}^{3} \frac{\partial \alpha_{j}}{\partial \widehat{\theta}}\right)\left(\dot{\hat{\theta}}-\tau_{i-1}\right),
\end{aligned}
$$

where $c_{j, i-1}$ is a positive design constant and $\tau_{i-1}=$ $\sum_{j=1}^{i-1} \gamma \varphi_{j}\left(\bar{x}_{j}, \widehat{\theta}\right) z_{j}^{4}$, then, there exists a virtual control law

$$
\begin{aligned}
\alpha_{i+1}\left(\bar{x}_{i}, \widehat{\theta}\right)= & -z_{i} \beta_{i}\left(\bar{x}_{i}, \widehat{\theta}\right), \\
\beta_{i}\left(\bar{x}_{i}, \widehat{\theta}\right)= & c_{i i}+\sum_{j=1}^{4} \phi_{i j}\left(\bar{x}_{i}, \widehat{\theta}\right) \\
& +\varphi_{i}\left(\bar{x}_{i}, \widehat{\theta}\right)\left(\widehat{\theta}-\gamma \sum_{j=2}^{i} z_{j}^{3} \frac{\partial \alpha_{j}}{\partial \widehat{\theta}}\right)+b_{i i},
\end{aligned}
$$

such that the ith Lyapunov-Krasovskii functional

$$
V_{i K}=V_{i-1, K}+\frac{1}{4} z_{i}^{4}+W_{i}, \quad W_{i}=\int_{t-d}^{t} \sum_{j=1}^{i} b_{i j} z_{j}^{4}(s) d s
$$

satisfies

$$
\begin{aligned}
\mathscr{L} V_{i K} \leq & -\sum_{j=1}^{i} c_{j i} z_{j}^{4}+z_{i}^{3}\left(x_{i+1}-\alpha_{i+1}\right) \\
& -\left(\frac{\tilde{\theta}}{\gamma}+\sum_{j=2}^{i} z_{j}^{3} \frac{\partial \alpha_{j}}{\partial \widehat{\theta}}\right)\left(\dot{\hat{\theta}}-\tau_{i}\right),
\end{aligned}
$$

where

$$
c_{j i}= \begin{cases}c_{j j}-\varepsilon_{j+1,1}-\sum_{k=2}^{4} \varepsilon_{i j k}-\sum_{k=5}^{6} \sum_{l=j}^{i-1} \varepsilon_{l+1, k}-\sum_{l=j}^{i-1} b_{l+1, j}, & j=1 ; \\ c_{j j}-\varepsilon_{j+1,1}-\sum_{k=2}^{4} \varepsilon_{i j k}-\sum_{l=j}^{i-1} b_{l+1, j}, & j=2, \ldots, i-1,\end{cases}
$$

and $b_{i j}, \varepsilon_{i j k}(k=2,3,4)$, and $\varepsilon_{i k}(k=1,5,6)$ are positive design constants; $\phi_{i j}(j=1, \ldots, 4), \varphi_{i}=\varphi_{i 1}+\varphi_{i 2}$, and $\varphi_{i 1}, \varphi_{i 2}$ are nonnegative continuous functions and $\tau_{i}=\tau_{i-1}+\gamma \varphi_{i} z_{i}^{4}$.

Proof. See Appendix.

Step $n$. By exactly following the design procedure at Step $i$, one can obtain the adaptive state-feedback controller

$$
\begin{aligned}
u(x, \widehat{\theta}) & =-z_{n} \beta_{n}(x, \widehat{\theta}), \\
\beta_{n}(x, \widehat{\theta}) & =c_{n n}+\sum_{i=1}^{4} \phi_{n i}(x, \widehat{\theta})
\end{aligned}
$$

$$
\begin{gathered}
+\varphi_{n}(x, \widehat{\theta})\left(\widehat{\theta}-\gamma \sum_{i=2}^{n} z_{i}^{3} \frac{\partial \alpha_{i}}{\partial \widehat{\theta}}\right)+b_{n n}, \\
\dot{\hat{\theta}}=\tau_{n}=\sum_{i=1}^{n} \gamma \varphi_{i}\left(\bar{x}_{i}, \widehat{\theta}\right) z_{i}^{4},
\end{gathered}
$$

which renders the Lyapunov-Krasovskii functional

$$
V_{n K}=\frac{1}{4} \sum_{i=1}^{n} z_{i}^{4}+\sum_{i=1}^{n} W_{i}+\frac{1}{2 \gamma} \widetilde{\theta}^{2}
$$


to satisfy

$$
\mathscr{L} V_{n K} \leq-\sum_{i=1}^{n} c_{i n} z_{i}^{4}
$$

where $\phi_{n i}$ and $\varphi_{n}$ are known nonnegative continuous functions; $b_{n n}, \varepsilon_{n 1}, \varepsilon_{n i 2}, \varepsilon_{n i 3}, \varepsilon_{n i 4}, \varepsilon_{n 5}, \varepsilon_{n 6}$, and $c_{i n}$ are positive design constants with

$$
c_{i n}= \begin{cases}c_{i i}-\varepsilon_{i+1,1}-\sum_{k=2}^{4} \varepsilon_{n i k}-\sum_{k=5}^{6} \sum_{l=i}^{n-1} \varepsilon_{l+1, k}-\sum_{l=i}^{n-1} b_{l+1, i}, & i=1 \\ c_{i i}-\varepsilon_{i+1,1}-\sum_{k=2}^{4} \varepsilon_{n i k}-\sum_{l=i}^{n-1} b_{l+1, i}, & i=2, \ldots, n-1 .\end{cases}
$$

\section{Stability Analysis}

We summarize the main result in the following theorem.

Theorem 8. For system (5), there exists an adaptive control law (21) such that (i) the closed-loop system consisting of (5), (9), (14), (17), and (21) is GAS in probability; (ii) $P\left\{\lim _{t \rightarrow \infty}|x(t)|=\right.$ $0\}=1$ and $P\left\{\lim _{t \rightarrow \infty} \widehat{\theta}(t)\right.$ exists and is finite $\}=1$.

Proof. In view of (23), it is obvious that $V_{n K}$ is $\mathscr{C}^{2}$ on $z=$ $\left(z_{1}, \ldots, z_{n}\right)^{T}$ and $\widehat{\theta}$. In addition, the inequality (4) in Lemma 2 is satisfied with $W(z)=\sum_{i=1}^{n} c_{i n} z_{i}^{4}$, which is a $\mathscr{K}$-class function with $c_{i n}>0$. In the sequel, we focus on verifying inequality (3) in Lemma 2.

On one hand, from (22) and $\left(x_{1}+\cdots+x_{n}\right)^{p} \leq$ $\max \left\{n^{p-1}, 1\right\}\left(x_{1}^{p}+\cdots+x_{n}^{p}\right)$, one has

$$
V_{n K} \geq \frac{1}{4} \sum_{i=1}^{n} z_{i}^{4} \geq \frac{1}{4 n}\left(\sqrt{\sum_{i=1}^{n} z_{i}^{2}}\right)^{4}
$$

Let $\chi_{1}(|z|, \widehat{\theta})=(1 / 4 n)|z|^{4}$; obviously, $\chi_{1}(|z|, \tilde{\theta}) \in \mathscr{K}_{\infty}$ and $\chi_{1}(|z|, \widetilde{\theta}) \leq V_{n K}(z, \widetilde{\theta})$ hold. On the other hand, by the mean value theorem, one can achieve

$$
\begin{aligned}
V_{n K}(z, \tilde{\theta}) \leq & \frac{1}{4} \sum_{i=1}^{n} z_{i}^{4}+d \sum_{i=1}^{n} W_{i}(z(\sigma))+\frac{1}{2 \gamma} \widetilde{\theta}^{2} \\
\leq & \frac{1}{4} \sum_{i=1}^{n} \sup _{d \leq s \leq 0} z_{i}^{4}(t+s)+d b \sum_{i=1}^{n} \sup _{d \leq s \leq 0} z_{i}^{4}(t+s) \\
& +\frac{1}{2 \gamma} \widetilde{\theta}^{2} \\
\leq & \left(\frac{1}{4}+d b\right)\left(\sqrt{\sum_{i=1}^{n} \sup _{-d \leq s \leq 0} z_{i}^{2}(t+s)}\right)^{4} \\
& +\frac{1}{2 \gamma} \widetilde{\theta}^{2},
\end{aligned}
$$

where $b=\max \left\{\sum_{j=1}^{i} b_{i j}, i=1, \ldots, n\right\}$ and $\sigma \in[t-d, t]$. Define $\chi_{2}\left(\sup _{-d \leq s \leq 0}|z(t+s)|, \widetilde{\theta}\right)=(1 / 4+d b)\left(\sup _{-d \leq s \leq 0}|z(t+s)|\right)^{4}+$ $(1 / 2 \gamma) \widetilde{\theta}^{2} \in \mathscr{K}_{\infty}$; then $V_{n K}(z, \widetilde{\theta}) \leq \chi_{2}\left(\sup _{-d \leq s \leq 0}|z(t+s)|, \widetilde{\theta}\right)$.
Hence, inequality (3) in Lemma 2 is satisfied. Thus, one concludes from Lemma 2 that (i) holds with $P\left\{\lim _{t \rightarrow \infty}|z(t)|=\right.$ $0\}=1$.

Furthermore, considering $\alpha_{i}(0, \widehat{\theta})=0(i=2, \ldots, n), u(0$, $\widehat{\theta})=0$, and (9), one further gets $P\left\{\lim _{t \rightarrow \infty}|x(t)|=0\right\}=1$. In addition, from (22)-(23), it holds that $\widetilde{\theta}(t)$ converges a.s. to a finite limit $\widetilde{\theta}_{\infty}$ as $t \rightarrow \infty$; that is, $P\left\{\lim _{t \rightarrow \infty} \widehat{\theta}(t)\right.$ exists and is finite $\}=1$. Hence, conclusion (ii) is proved, which completes the proof of Theorem 8 .

Remark 9. We emphasize two main points. (i) For system (5), this paper completely removes the growth assumptions imposed on system time-delay nonlinearities. (ii) The construction of adaptive controller (21) is difficult and the proof of Theorem 8 is not a trivial work.

\section{A Simulation Example}

In this section, we give a simulation example to verify the proposed scheme in Section 3.

Example 1. Consider stochastic nonlinear time-delay system

$$
\begin{aligned}
d x_{1}= & x_{2} d t+\left(x_{1}^{3}+x_{1}^{2} \sin \left(x_{1}(t-d)\right)\right) d t \\
& +\frac{1}{2} x_{1}^{2} \Sigma \mathrm{d} \omega \\
d x_{2}= & u d t+\left(x_{1}(t-d)+x_{2}(t-d)\right) d t+x_{1} \Sigma d \omega
\end{aligned}
$$

where $\Sigma$ is defined as in (5) and $d>0$ is a time delay. It can be verified that $\rho_{11}=x_{1}^{2}, \rho_{21}=1, \rho_{22}=1, \bar{g}_{1}=x_{1} / 2$, and $\bar{g}_{2}=1$ in Lemma 5 and (8).

Then, by exactly following the design procedure in Section 3 , one can get the adaptive controller with the form

$$
\begin{aligned}
& z_{1}=x_{1}, \\
& z_{2}=x_{2}-\alpha_{2}\left(x_{1}, \widehat{\theta}\right), \\
& \alpha_{2}\left(x_{1}, \widehat{\theta}\right)=-z_{1} \beta_{1}\left(x_{1}, \widehat{\theta}\right), \\
& \beta_{1}=c_{11}+\rho_{11}\left(x_{1}\right)+\phi_{11}\left(x_{1}\right)+\varphi_{1}\left(x_{1}\right) \hat{\theta}+b_{11},
\end{aligned}
$$




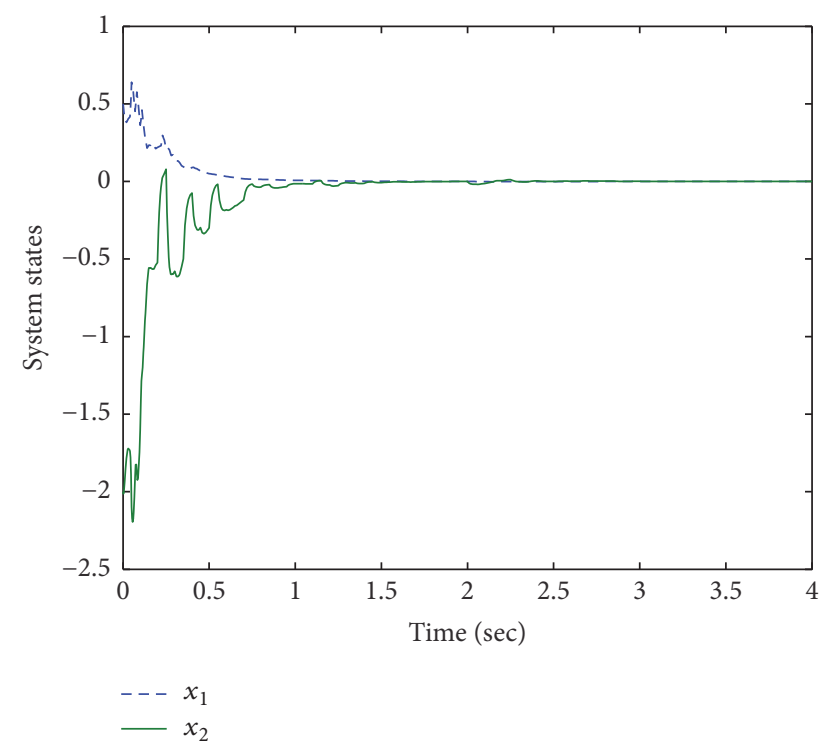

FIgURE 1: The state responses of the closed-loop system (27)-(28).

$$
\begin{aligned}
& u\left(\bar{x}_{2}, \hat{\theta}\right)=-z_{2}\left(c_{22}+\sum_{i=1}^{4} \phi_{2 i}\left(\bar{x}_{2}, \hat{\theta}\right)\right. \\
& \left.+\varphi_{2}\left(\bar{x}_{2}, \widehat{\theta}\right)\left(\hat{\theta}-\gamma z_{2}^{3} \frac{\partial \alpha_{2}}{\partial \widehat{\theta}}\right)+b_{22}\right), \\
& \dot{\hat{\theta}}=\gamma \varphi_{1}\left(x_{1}\right) z_{1}^{4}+\gamma \varphi_{2}\left(\bar{x}_{2}, \hat{\theta}\right) z_{2}^{4},
\end{aligned}
$$

where $b_{11}, b_{21}, b_{22}, c_{11}, c_{22}, \varepsilon_{21}, \varepsilon_{212}, \varepsilon_{213}, \varepsilon_{214}, \varepsilon_{25}, \varepsilon_{26}$, and $\gamma$ are positive design constants; $\phi_{11}=(3 / 4)\left(4 b_{11}\right)^{-1 / 3} \rho_{11}^{4 / 3}$; $\varphi_{1}=(3 / 2)\left|\bar{g}_{1}\right|^{2} ; \phi_{21}=(1 / 4)\left((4 / 3) \varepsilon_{21}\right)^{-3} ; \phi_{22}=\widetilde{l}_{22}+$ $(3 / 4)\left(4 \varepsilon_{212}\right)^{-1 / 3} \widetilde{l}_{21}^{4 / 3}+\sum_{i=1}^{2}(3 / 4)\left(4 b_{2 i}\right)^{-1 / 3} \widetilde{l}_{2 i}^{4 / 3}$ with $s_{1}=$ $\max \left\{1,\left|\beta_{1}\right|\right\}, l_{21}=\rho_{21}+\rho_{22} s_{1}, l_{22}=\rho_{22} s_{1}, l_{11}=\rho_{11}$, $\tilde{l}_{21}=\left(l_{11}+l_{21}\right) \max \left\{1,\left|\partial \alpha_{2} / \partial x_{1}\right|\right\}, \widetilde{l}_{22}=l_{22} \max \left\{1,\left|\partial \alpha_{2} / \partial x_{1}\right|\right\}$; $\phi_{23}=\bar{\phi}_{23}+(3 / 4)\left(4 \varepsilon_{213}\right)^{-1 / 3} \bar{\phi}_{13}^{4 / 3}$ with $\bar{\phi}_{13}=\bar{\phi}_{23}=\left|\partial \alpha_{2} / \partial x_{1}\right| s_{1}$; $\phi_{24}=(3 / 4)\left(4 \varepsilon_{214}\right)^{-1 / 3}\left(\left|\partial \alpha_{2} / \partial \widehat{\theta}\right| \bar{\varphi}_{1}\right)^{4 / 3}$ with $\bar{\varphi}_{1}=\gamma \varphi_{1} z_{1}^{3}$; $\varphi_{21}=(3 / 4)\left(4 \varepsilon_{25}\right)^{-1 / 3}\left((1 / 2)\left|\partial^{2} \alpha_{2} / \partial x_{1}^{2}\right|\left|\bar{g}_{1}\right|^{2}\left|z_{1}\right|\right)^{4 / 3} ; \varphi_{22}=$ $(1 / 2)\left(2 \varepsilon_{26}\right)^{-1}\left((3 / 2)\left|\bar{g}_{2}-\left(\partial \alpha_{2} / \partial x_{1}\right) \bar{g}_{1}\right|^{2}\right)^{2}$ and $\varphi_{2}=\varphi_{21}+\varphi_{22}$ are all nonnegative continuous functions.

In simulation, choose $b_{11}=1, b_{21}=1, b_{22}=1, c_{11}=3$, $c_{22}=1, \varepsilon_{21}=0.1, \varepsilon_{212}=0.1, \varepsilon_{213}=0.1, \varepsilon_{214}=0.1, \varepsilon_{25}=0.1$, $\varepsilon_{26}=0.1, \gamma=5, d=2$, and $\Sigma=1$. The initial values are given by $x_{1}(0)=0.5, x_{2}(0)=-2$, and $\hat{\theta}(0)=1$. Figures $1-3$ demonstrate the effectiveness of the control scheme.

\section{Conclusions}

This note solves the adaptive state-feedback control for stochastic nonlinear time-delay systems driven by unknown covariance noise. The traditional assumptions imposed on system nonlinearities are removed and the negative effects generated by unknown covariance noise are eliminated by

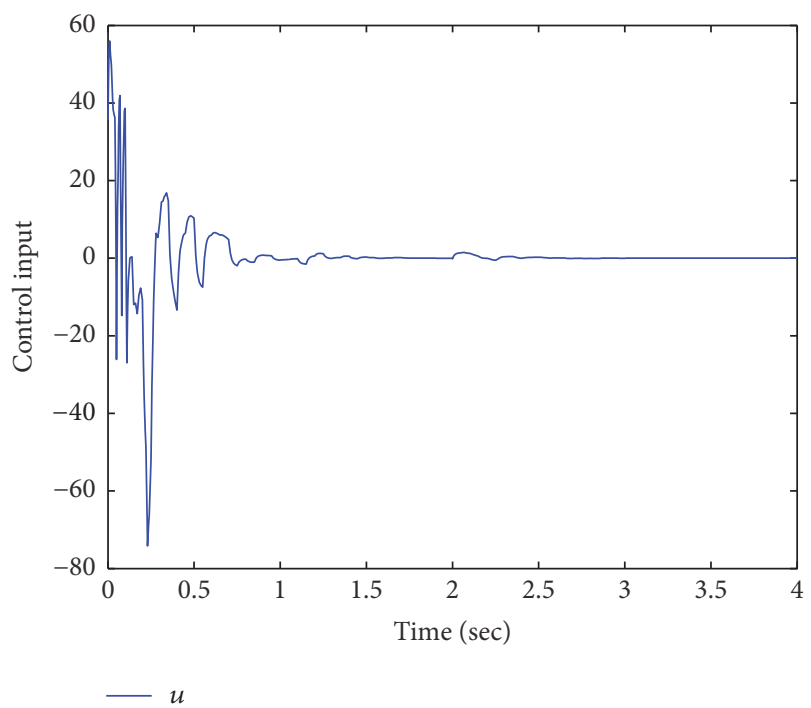

FIGURE 2: The control input responses of the closed-loop system (27)-(28).

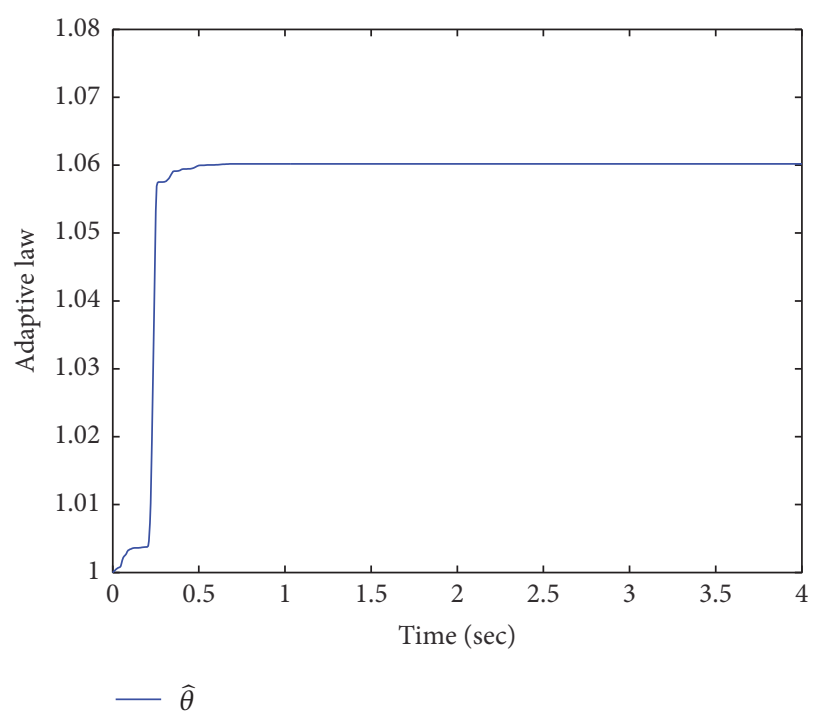

FIgURE 3: Adaptive control law of the closed-loop system (27)-(28).

using Lyapunov-functionals and adaptive backstepping technique. In addition, an adaptive state-feedback controller is designed to enable the closed-loop system to be GAS in probability. One more problem under investigation is how to solve the output-feedback control problem for system (5).

\section{Appendix}

Proof of Proposition 7. Firstly, in terms of (2), (11), (16), and (18), one arrives at

$$
\begin{aligned}
\mathscr{L} V_{i K} \leq & -\sum_{j=1}^{i-1} c_{j, i-1} z_{j}^{4}+z_{i}^{3} x_{i+1}+z_{i-1}^{3}\left(x_{i}-\alpha_{i}\right)+z_{i}^{3} F_{i d} \\
& -z_{i}^{3} \sum_{j=1}^{i-1} \frac{\partial \alpha_{i}}{\partial x_{j}} x_{j+1}-z_{i}^{3} \frac{\partial \alpha_{i}}{\partial \widehat{\theta}} \tau_{i-1}
\end{aligned}
$$




$$
\begin{aligned}
& -\frac{1}{2} z_{i}^{3} \sum_{j, k=1}^{i-1} \frac{\partial^{2} \alpha_{i}}{\partial x_{j} \partial x_{k}} g_{j} \Sigma \Sigma^{T} g_{k}^{T} \\
& +\frac{3}{2} z_{i}^{2} G_{i} \Sigma \Sigma^{T} G_{i}^{T}+\sum_{j=1}^{i-1} b_{i j} z_{j}^{4}+b_{i i} z_{i}^{4} \\
& -\sum_{j=1}^{i} b_{i j} z_{j}^{4}(t-d) \\
& -\left(\frac{\tilde{\theta}}{\gamma}+\sum_{j=2}^{i} z_{j}^{3} \frac{\partial \alpha_{j}}{\partial \widehat{\theta}}\right)\left(\dot{\hat{\theta}}-\tau_{i-1}\right) .
\end{aligned}
$$

To proceed further, we try to estimate the third-eighth terms in the right-hand side of (A.1).

According to (9), one has

$$
\left|x_{i}\right|=\left|z_{i}-\beta_{i-1} z_{i-1}\right| \leq s_{i-1}\left(\left|z_{i}\right|+\left|z_{i-1}\right|\right),
$$

where $s_{i-1}=\max \left\{1,\left|\beta_{i-1}\right|\right\}$. Using (A.2) and Lemmas 4 and 5 , it is easy to verify that

$$
\begin{array}{r}
\left|f_{i d}\right| \leq \sum_{j=1}^{i} \rho_{i j}\left(\bar{x}_{j}\right)\left(s_{j-1}\left(\left|z_{j-1}\right|+\left|z_{j}\right|\right)\right. \\
\left.+s_{j-1}\left(\left|z_{j-1}(t-d)\right|+\left|z_{j}(t-d)\right|\right)\right) \\
\leq \sum_{j=1}^{i} l_{i j}\left(\bar{x}_{j}, \hat{\theta}\right)\left(\left|z_{j}\right|+\left|z_{j}(t-d)\right|\right),
\end{array}
$$

where

$$
l_{i j}\left(\bar{x}_{j}, \hat{\theta}\right)= \begin{cases}\rho_{i, j+1} s_{j}+\rho_{i j} s_{j-1}, & j=1, \ldots, i-1, \\ \rho_{i j} s_{j-1}, & j=i\end{cases}
$$

with $s_{0}=1$. Now, we turn to give the estimate procedure. By applying (9)-(10), (A.2)-(A.3), and Lemma 4, it can be verified that

$$
\begin{aligned}
& z_{i-1}^{3}\left(x_{i}-\alpha_{i}\right)=z_{i-1}^{3}\left(z_{i}+\alpha_{i}-\alpha_{i}\right) \leq \varepsilon_{i 1} z_{i-1}^{4}+\phi_{i 1} z_{i}^{4}, \\
& z_{i}^{3} F_{i d} \leq\left|z_{i}\right|^{3}\left|f_{i d}-\sum_{j=1}^{i-1} \frac{\partial \alpha_{i}}{\partial x_{j}} f_{j d}\right| \\
& \leq\left|z_{i}\right|^{3} \sum_{j=1}^{i} \widetilde{l}_{i j}\left(\bar{x}_{j}, \widehat{\theta}\right)\left(\left|z_{j}\right|+\left|z_{j}(t-d)\right|\right) \\
& \leq \sum_{j=1}^{i-1} \varepsilon_{i j 2} z_{j}^{4}+\phi_{i 2}\left(\bar{x}_{i}, \widehat{\theta}\right) z_{i}^{4}+\sum_{j=1}^{i} b_{i j} z_{j}^{4}(t-d), \\
& -z_{i}^{3} \sum_{j=1}^{i-1} \frac{\partial \alpha_{i}}{\partial x_{j}} x_{j+1} \leq\left|z_{i}\right|^{3} \sum_{j=1}^{i-1}\left|\frac{\partial \alpha_{i}}{\partial x_{j}}\right| s_{j}\left(\left|z_{j}\right|+\left|z_{j+1}\right|\right)
\end{aligned}
$$

$$
\begin{aligned}
& \quad \leq\left|z_{i}\right|^{3} \sum_{j=1}^{i-1} \bar{\phi}_{j 3}\left(\bar{x}_{j}, \widehat{\theta}\right)\left|z_{j}\right| \\
& \leq \sum_{j=1}^{i-1} \varepsilon_{i j 3} z_{j}^{4}+\phi_{i 3}\left(\bar{x}_{i}, \widehat{\theta}\right) z_{i}^{4}, \\
& -z_{i}^{3} \frac{\partial \alpha_{i}}{\partial \widehat{\theta}} \tau_{i-1} \leq\left|z_{i}\right|^{3}\left|\frac{\partial \alpha_{i}}{\partial \widehat{\theta}}\right| \sum_{j=1}^{i-1} \bar{\varphi}_{j}\left(\bar{x}_{j}, \widehat{\theta}\right) z_{j} \\
& \leq \sum_{j=1}^{i-1} \varepsilon_{i j 4} z_{j}^{4}+\phi_{i 4}\left(\bar{x}_{i}, \widehat{\theta}\right) z_{i}^{4}, \\
& -\frac{1}{2} z_{i}^{3} \sum_{j, k=1}^{i-1} \frac{\partial^{2} \alpha_{i}}{\partial x_{j} \partial x_{k}} g_{j} \Sigma \Sigma^{T} g_{k}^{T} \\
& \leq \frac{1}{2}\left|z_{i}\right|^{3} \sum_{j, k=1}^{i-1}\left|\frac{\partial^{2} \alpha_{i}}{\partial x_{j} \partial x_{k}}\right|\left|\bar{g}_{j} \bar{g}_{k}\right|\left|\Sigma \Sigma^{T}\right| z_{1}^{2} \\
& \leq \varepsilon_{i 5} z_{1}^{4}+\varphi_{i 1}\left(\bar{x}_{i}, \widehat{\theta}\right) z_{i}^{4}\left|\Sigma \Sigma^{T}\right|^{4 / 3} \\
& \quad \leq \varepsilon_{i 5} z_{1}^{4}+\varphi_{i 1}\left(\bar{x}_{i}, \widehat{\theta}\right) z_{i}^{4} \theta \\
& \quad \leq \varepsilon_{i 6} z_{1}^{4}+\varphi_{i 2}\left(\bar{x}_{i}, \widehat{\theta}\right) z_{i}^{4}\left|\Sigma \Sigma^{T}\right|^{2} \\
& \quad \leq \varepsilon_{i 6} z_{1}^{4}+\varphi_{i 2}\left(\bar{x}_{i}, \widehat{\theta}\right) z_{i}^{4} \theta \\
& \frac{3}{2} z_{i}^{2} G_{i} \Sigma \Sigma^{T} G_{i}^{T} \leq \frac{3}{2} z_{i}^{2}\left|\bar{g}_{i}-\sum_{j=1}^{i-1} \frac{\partial \alpha_{i}}{\partial x_{j}} \bar{g}_{j}\right|^{2} z_{1}^{2}\left|\Sigma \Sigma^{T}\right|
\end{aligned}
$$

where $b_{i j}, \varepsilon_{i 1}, \varepsilon_{i j 2}, \varepsilon_{i j 3}, \varepsilon_{i j 4}, \varepsilon_{i 5}$, and $\varepsilon_{i 6}$ are positive design constants; $\phi_{i 1}=(1 / 4)\left((4 / 3) \varepsilon_{i 1}\right)^{-3} ; \widetilde{l}_{i j}=\sum_{k=j}^{i} l_{k j} \max \left\{1,\left|\partial \alpha_{i} / \partial x_{j}\right|\right\}$, $\phi_{i 2}=\widetilde{l}_{i i}+\sum_{j=1}^{i-1}(3 / 4)\left(4 \varepsilon_{i j 2}\right)^{-1 / 3} \widetilde{l}_{i j}^{4 / 3}+\sum_{j=1}^{i}(3 / 4)\left(4 b_{i j}\right)^{-1 / 3} \widetilde{l}_{i j}^{4 / 3}$; $\bar{\phi}_{j 3}=\left|\partial \alpha_{i} / \partial x_{j}\right| s_{j}$ for $j=1, \bar{\phi}_{j 3}=\left|\partial \alpha_{i} / \partial x_{j-1}\right| s_{j-1}+\left|\partial \alpha_{i} / \partial x_{j}\right| s_{j}$ for $j=2, \ldots, i-1$ and $\bar{\phi}_{j 3}=\left|\partial \alpha_{i} / \partial x_{j-1}\right| s_{j-1}$ for $j=$ $i ; \phi_{i 3}=\bar{\phi}_{i 3}+\sum_{j=1}^{i-1}(3 / 4)\left(4 \varepsilon_{i j 3}\right)^{-1 / 3}\left|\bar{\phi}_{j 3}\right|^{4 / 3} ; \bar{\varphi}_{j}=\gamma \varphi_{j}\left|z_{j}\right|^{3} ;$ $\varphi_{i 1}=(3 / 4)\left(4 \varepsilon_{i 5}\right)^{-1 / 3}\left((1 / 2) \sum_{j, k=1}^{i-1}\left|\partial^{2} \alpha_{i} / \partial x_{j} \partial x_{k}\right|\left|\bar{g}_{j} \bar{g}_{k}\right|\left|z_{1}\right|\right)^{4 / 3} ;$ $\varphi_{i 2}=(1 / 4)\left(\varepsilon_{i 6}\right)^{-1}\left((3 / 2)\left|\bar{g}_{i}-\sum_{j=1}^{i-1}\left(\partial \alpha_{i} / \partial x_{j}\right) \bar{g}_{j}\right|^{2}\right)^{2}$ and $\phi_{i 4}=$ $\sum_{j=1}^{i-1}(3 / 4)\left(4 \varepsilon_{i j 4}\right)^{-1 / 3}\left(\left|\partial \alpha_{i} / \partial \widehat{\theta}\right| \bar{\varphi}_{j}\right)^{4 / 3}$.

Then, substituting (A.5) into (A.1) yields

$$
\begin{aligned}
\mathscr{L} V_{i K} \leq & -\sum_{j=1}^{i-1} c_{j, i-1} z_{j}^{4}+\left(\varepsilon_{i 5}+\varepsilon_{i 6}\right) z_{1}^{4}+\varepsilon_{i 1}\left|z_{i-1}\right|^{4} \\
& +\sum_{k=2}^{4} \sum_{j=1}^{i-1} \varepsilon_{i j k}\left|z_{j}\right|^{4}+\sum_{j=1}^{i-1} b_{i j} z_{j}^{4} \\
& +z_{i}^{3}\left(x_{i+1}-\alpha_{i+1}\right)+z_{i}^{3} \alpha_{i+1} \\
& +z_{i}^{4}\left(\sum_{j=1}^{4} \phi_{i j}\left(\bar{x}_{i}, \widehat{\theta}\right)+\varphi_{i}\left(\bar{x}_{i}, \hat{\theta}\right) \hat{\theta}+b_{i i}\right)
\end{aligned}
$$




$$
\begin{aligned}
& +\varphi_{i}\left(\bar{x}_{i}, \widehat{\theta}\right) z_{i}^{4} \widetilde{\theta} \\
& -\left(\frac{\tilde{\theta}}{\gamma}+\sum_{j=2}^{i} z_{j}^{3} \frac{\partial \alpha_{j}}{\partial \widehat{\theta}}\right)\left(\dot{\hat{\theta}}-\tau_{i-1}\right),
\end{aligned}
$$

where $\varphi_{i}=\varphi_{i 1}+\varphi_{i 2}$. Hence, by choosing $\alpha_{i+1}$ as (17), $c_{j i}$ as (20), and $\tau_{i}=\tau_{i-1}+\gamma \varphi_{i} z_{i}^{4}$, one can finally get (19). This completes the proof.

\section{Conflicts of Interest}

The authors declare that they have no conflicts of interest.

\section{Acknowledgments}

This work is supported by National Natural Science Foundation of China (nos. 61573172, 61503166), 333 High-Level Talents Training Program in Jiangsu Province (no. BRA2015352), Program for Fundamental Research of Natural Sciences in Universities of Jiangsu Province (no. 15KJB510011), and Shandong Province Natural Science Foundation of China (no. ZR2016AL05).

\section{References}

[1] H. Deng and M. Krstić, "Stochastic nonlinear stabilization-I: a backstepping design," Systems and Control Letters, vol. 32, no. 3, pp. 143-150, 1997.

[2] Z. Pan and T. Başar, "Backstepping controller design for nonlinear stochastic systems under a risk-sensitive cost criterion," SIAM Journal on Control and Optimization, vol. 37, no. 3, pp. 957-995, 1999.

[3] X. R. Mao, Stochastic Differential Equations and Their Applications, Horwood Publishing, Chichester, UK, 2007.

[4] X.-J. Xie and N. Duan, "Output tracking of high-order stochastic nonlinear systems with application to benchmark mechanical system," IEEE Transactions on Automatic Control, vol. 55, no. 5, pp. 1197-1202, 2010.

[5] F. Li and Y. Liu, "General stochastic convergence theorem and stochastic adaptive output-feedback controller," IEEE Transactions on Automatic Control, 2016.

[6] X.-J. Xie, N. Duan, and C.-R. Zhao, "A combined homogeneous domination and sign function approach to output-feedback stabilization of stochastic high-order nonlinear systems," IEEE Transactions on Automatic Control, vol. 59, no. 5, pp. 1303-1309, 2014.

[7] S. Khoo, J. Yin, Z. Man, and X. Yu, "Finite-time stabilization of stochastic nonlinear systems in strict-feedback form," Automatica, vol. 49, no. 5, pp. 1403-1410, 2013.

[8] J. Yin, S. Khoo, and Z. Man, "Finite-time stability theorems of homogeneous stochastic nonlinear systems," Systems \& Control Letters, vol. 100, pp. 6-13, 2017.

[9] Z.-J. Wu, X.-J. Xie, and S.-Y. Zhang, "Stochastic adaptive backstepping controller design by introducing dynamic signal and changing supply function," International Journal of Control, vol. 79, no. 12, pp. 1635-1646, 2006.

[10] X.-J. Xie and J. Tian, "Adaptive state-feedback stabilization of high-order stochastic systems with nonlinear parameterization," Automatica, vol. 45, no. 1, pp. 126-133, 2009.
[11] N. Duan and H.-F. Min, "Decentralized adaptive NN statefeedback control for large-scale stochastic high-order nonlinear systems," Neurocomputing, vol. 173, no. 3, pp. 1412-1421, 2016.

[12] H.-F. Min and N. Duan, "NN-based output-feedback control for stochastic nonlinear systems with unknown control directions," Asian Journal of Control, vol. 18, no. 6, pp. 1-10, 2016.

[13] Y. Xia, M. Fu, P. Shi, Z. Wu, and J. Zhang, "Adaptive backstepping controller design for stochastic jump systems," IEEE Transactions on Automatic Control, vol. 54, no. 12, pp. 28532859, 2009.

[14] N. Duan, H. Min, and H. Chu, "A homogeneous domination approach to partial-state-feedback control for stochastic highorder nonlinear systems," in Proceedings of the 28th Chinese Control and Decision Conference (CCDC '16), pp. 1813-1818, Yinchuan, China, May 2016.

[15] H.-F. Min and N. Duan, "Adaptive output-feedback control for stochastic nonlinear systems using neural networks," in Proceedings of the 33rd Chinese Control Conference (CCC '14), pp. 5288-5293, IEEE, Nanjing, China, July 2014.

[16] H.-B. Ji and H.-S. Xi, "Adaptive output-feedback tracking of stochastic nonlinear systems," IEEE Transactions on Automatic Control, vol. 51, no. 2, pp. 355-360, 2006.

[17] Z.-J. Wu, X.-J. Xie, and S.-Y. Zhang, "Adaptive backstepping controller design using stochastic small-gain theorem," Automatica, vol. 43, no. 4, pp. 608-620, 2007.

[18] Y. Fu, Z. Tian, and S. Shi, "Output feedback stabilization for a class of stochastic time-delay nonlinear systems," IEEE Transactions on Automatic Control, vol. 50, no. 6, pp. 847-851, 2005.

[19] L. Liu, X. Li, H. Wang, and B. Niu, "Global asymptotic stabilization of stochastic feedforward nonlinear systems with input time-delay," Nonlinear Dynamics, vol. 83, no. 3, pp. 15031510, 2016.

[20] L. Liu, X.-D. Zhao, B. Niu, H.-Q. Wang, and X.-J. Xie, "Global output-feedback stabilisation of switched stochastic non-linear time-delay systems under arbitrary switchings," IET Control Theory \& Applications, vol. 9, no. 2, pp. 283-292, 2015.

[21] T. Jiao, S. Xu, J. Lu, Y. Wei, and Y. Zou, "Decentralised adaptive output feedback stabilisation for stochastic time-delay systems via LaSalle-Yoshizawa-type theorem," International Journal of Control, vol. 89, no. 1, pp. 69-83, 2016.

[22] S.-J. Liu, S. S. Ge, and J.-F. Zhang, "Adaptive output-feedback control for a class of uncertain stochastic non-linear systems with time delays," International Journal of Control, vol. 81, no. 8, pp. 1210-1220, 2008.

[23] L. Liu and X.-J. Xie, "Output-feedback stabilization for stochastic high-order nonlinear systems with time-varying delay," Automatica, vol. 47, no. 12, pp. 2772-2779, 2011.

[24] W. Chen, J. Wu, and L. C. Jiao, "State-feedback stabilization for a class of stochastic time-delay nonlinear systems," International Journal of Robust and Nonlinear Control, vol. 22, no. 17, pp. 19211937, 2012.

[25] X.-J. Xie and L. Liu, "A homogeneous domination approach to state feedback of stochastic high-order nonlinear systems with time-varying delay," IEEE Transactions on Automatic Control, vol. 58, no. 2, pp. 494-499, 2013.

[26] W. Zha, J. Zhai, and S. Fei, “Output feedback control for a class of stochastic high-order nonlinear systems with time-varying delays," International Journal of Robust and Nonlinear Control, vol. 24, no. 16, pp. 2243-2260, 2014. 
[27] X.-J. Xie and L. Liu, "Further results on output feedback stabilization for stochastic high-order nonlinear systems with time-varying delay," Automatica, vol. 48, no. 10, pp. 2577-2586, 2012.

[28] H. Min and N. Duan, "Adaptive NN state-feedback control for stochastic high-order nonlinear systems with time-varying control direction and delays," Mathematical Problems in Engineering, vol. 2015, Article ID 723425, 11 pages, 2015.

[29] H. F. Min and N. Duan, "Adaptive NN output-feedback control for stochastic time-delay nonlinear systems with unknown control directions and perturbations," Nonlinear Analysis: Modeling and Control, vol. 21, no. 4, pp. 515-530, 2016.

[30] Z. X. Yu and S. G. Li, "Neural-network-based output-feedback adaptive dynamic surface control for a class of stochastic nonlinear time-delay systems with unknown control directions," Neurocomputing, vol. 129, pp. 540-547, 2014.

[31] G. Cui, T. Jiao, Y. Wei, G. Song, and Y. Chu, "Adaptive neural control of stochastic nonlinear systems with multiple timevarying delays and input saturation," Neural Computing and Applications, vol. 25, no. 3-4, pp. 779-791, 2014.

[32] T. S. Li, Z. F. Li, D. Wang, and C. L. P. Chen, "Outputfeedback adaptive neural control for stochastic nonlinear timevarying delay systems with unknown control directions," IEEE Transactions on Neural Networks and Learning Systems, vol. 26, no. 6, pp. 1188-1201, 2015.

[33] L. Liu, S. Yin, L. Zhang, X. Yin, and H. Yan, "Improved results on asymptotic stabilization for stochastic nonlinear time-delay systems with application to a chemical reactor system," IEEE Transactions on Systems, Man, and Cybernetics: Systems, vol. 47, no. 1, pp. 195-204, 2017.

[34] H. Deng, M. Krstic', and R. J. Williams, "Stabilization of stochastic nonlinear systems driven by noise of unknown covariance," IEEE Transactions on Automatic Control, vol. 46, no. 8, pp. 12371253, 2001.

[35] H. E. Psillakis and A. T. Alexandridis, "NN-based adaptive tracking control of uncertain nonlinear systems disturbed by unknown covariance noise," IEEE Transactions on Neural Networks, vol. 18, no. 6, pp. 1830-1835, 2007.

[36] C.-R. Zhao, X.-J. Xie, and N. Duan, "Adaptive state-feedback stabilization for high-order stochastic nonlinear systems driven by noise of unknown covariance," Mathematical Problems in Engineering, vol. 2012, Article ID 246579, 13 pages, 2012.

[37] W. Lin and C. Qian, "Adding one power integrator: a tool for global stabilization of high-order lower-triangular systems," Systems and Control Letters, vol. 39, no. 5, pp. 339-351, 2000.

[38] X. Zhang, W. Lin, and Y. Lin, "Nonsmooth feedback control of time-delay nonlinear systems: a dynamic gain based approach," IEEE Transactions on Automatic Control, vol. 62, no. 1, pp. 438444, 2017. 


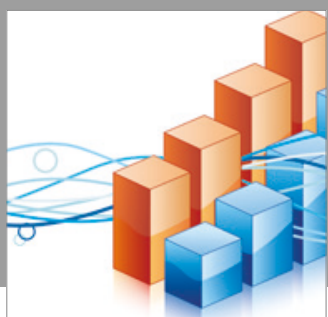

Advances in

Operations Research

vatersals

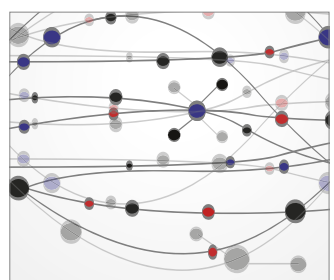

\section{The Scientific} World Journal
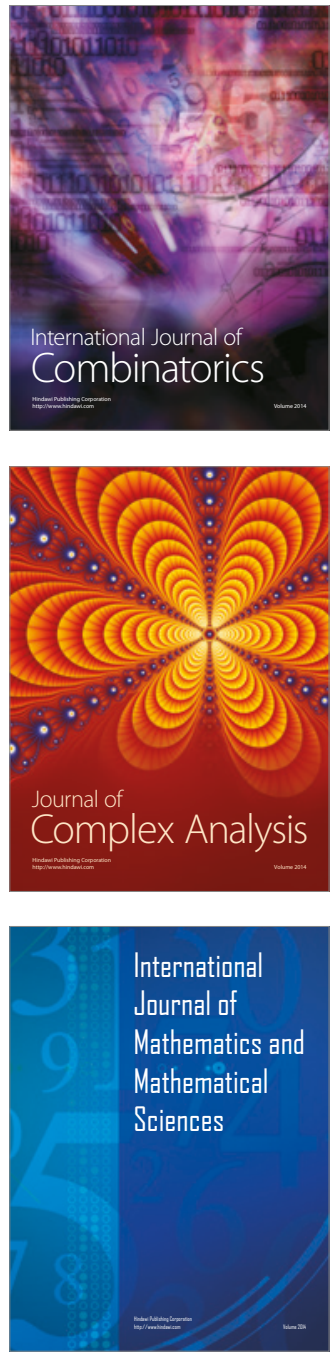
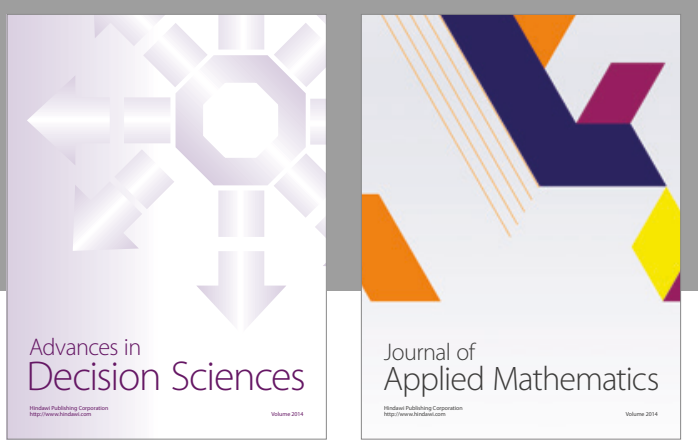

Algebra

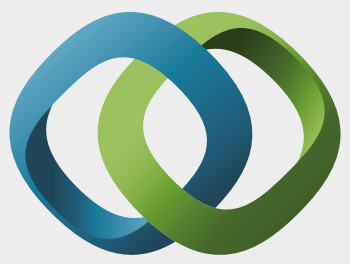

\section{Hindawi}

Submit your manuscripts at

https://www.hindawi.com
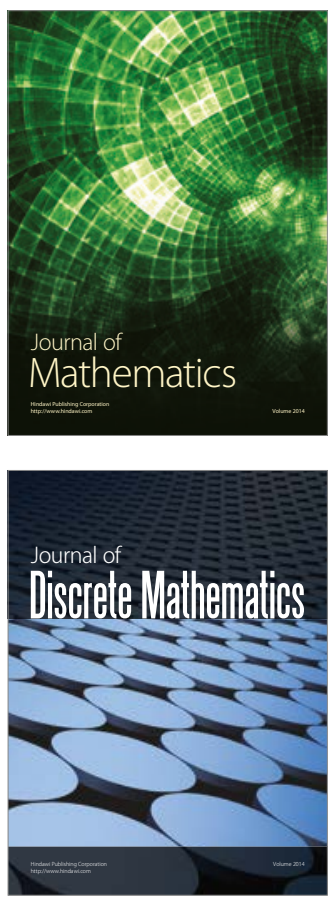

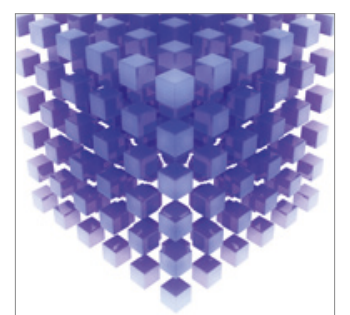

Mathematical Problems in Engineering
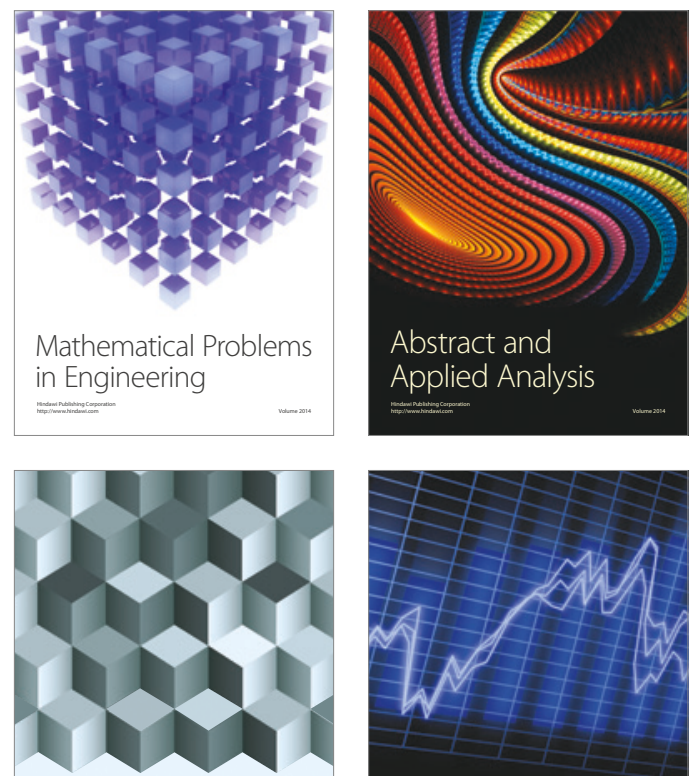

Journal of

Function Spaces

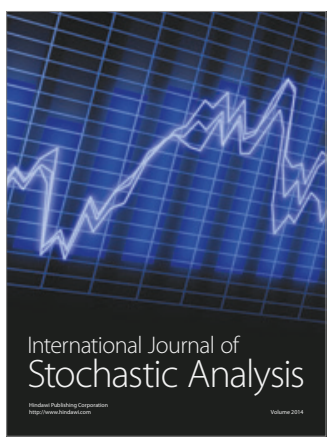

Probability and Statistics
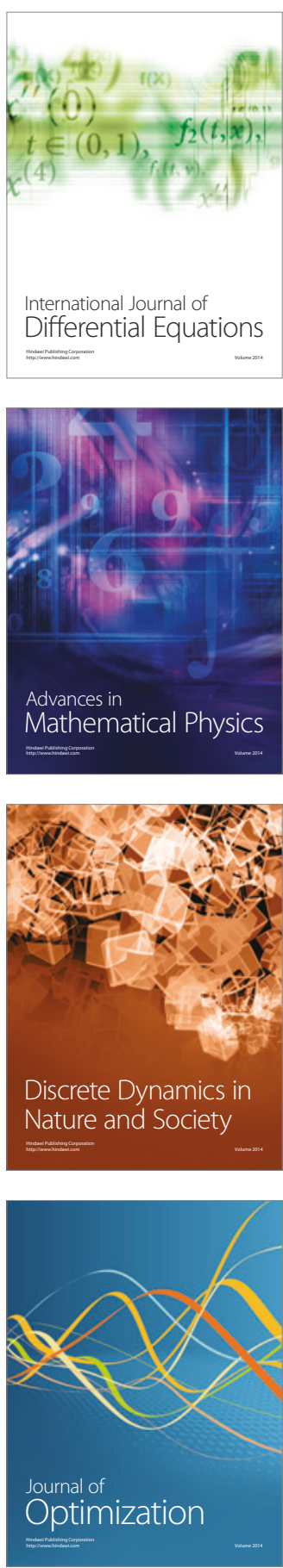